\title{
Universal Description of Granular Metals at Low Temperatures: Granular Fermi Liquid
}

\author{
I. S. Beloborodov, A. V. Lopatin, and V. M. Vinokur \\ Materials Science Division, Argonne National Laboratory, Argonne, Illinois 60439
}

(Dated: November 20, 2018)

\begin{abstract}
We present a unified description of the low temperature phase of granular metals that reveals a striking generality of the low temperature behaviors. Our model explains the universality of the low-temperature conductivity that coincides exactly with that of the homogeneously disordered systems and enables a straightforward derivation of low temperature characteristics of disordered conductors.

PACS numbers: $73.23 \mathrm{Hk}, 73.22 \mathrm{Lp}, 71.30 .+\mathrm{h}$
\end{abstract}

Granular metals exhibit a wealth of behaviors generic to strongly interacting disordered electronic systems and offer a unique experimental tool for studying the interplay between the effects of disorder and interactions. Depending on the strength of coupling between the grains these systems can assume either insulating- or metallic phases. Remarkably, metallic samples exhibit qualitatively different transport properties in different temperature regimes; in particular, the low temperature phase appears to be similar to disordered Fermi liquids.

The electronic transport in granular metals is governed by the nontrivial interplay between the diffusive intragrain electron motion and grain-to-grain tunneling which is accompanied by sequential charging of the grains involved in the particular electron transfer process. This brings the notion of the Coulomb blockade, and one expects that it is the competition of inter-grain coupling and electron-electron Coulomb interactions that eventually controls transport properties of granular metals. The basic parameter that characterizes transport properties is the dimensionless tunneling conductance, $g_{T}$. Depending on the bare tunneling conductance $g_{T}^{(0)}$, the conductivity can demonstrate either exponential (insulating)-, at $g_{T}^{(0)} \ll g_{T}^{C}$, or logarithmic (metallic), at $g_{T}^{(0)} \gg g_{T}^{C}$, temperature dependencies 1, 2, 3, 4], experiencing metalinsulator transition at $g_{T}^{(0)}=g_{T}^{C}$.

The metallic phase was recently studied in Ref. 1 where it was shown that the low temperature dependence of the conductivity of granular metals coincides exactly with the corresponding result for the conductivity of the homogeneously disordered samples. A question immediately arises: is it a coincidence that two different physical systems exhibit identical low temperature transport behaviors, or there is an underlying deep connection between the two? Furthermore, do all the other physical quantities (specific heat, tunneling density of states, etc.) possess the same universality? The main result of our Letter is the answer to these fundamental questions.

Generally speaking, all the universalities that one observes in nature can usually be attributed to one or another kind of the fundamental symmetry inherent to the physical system in question. For example, all critical phenomena are described in terms of universal models (Ginzburg - Landau Hamiltonian) that essentially include only the information about the large scale symmetry of the order parameter corresponding to the relevant degrees of freedom. Thus in order to uncover the universality of inhomogeneous metals one has to seek for a universal description in terms of the appropriate large scale degrees of freedom that characterize disordered conductors.

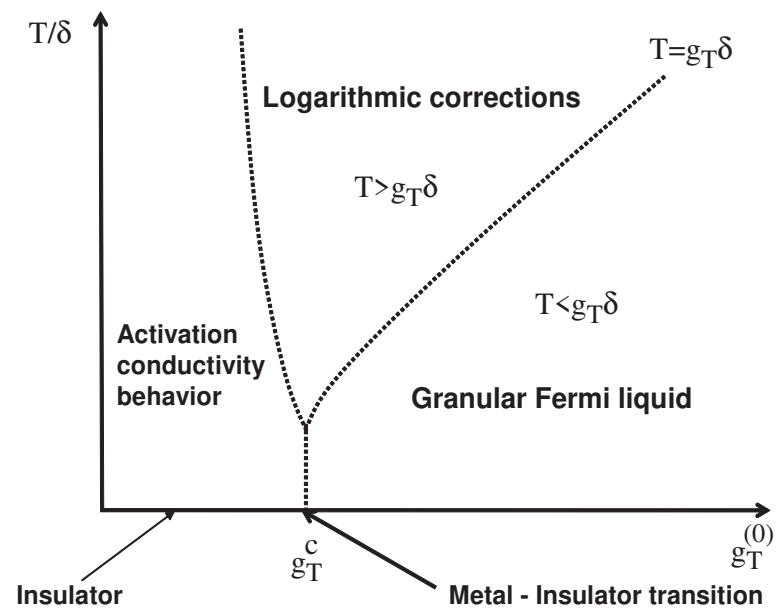

FIG. 1: Schematic phase diagram for granular metals showing crossovers between three distinct phases. At small bare tunneling conductances, $g_{T}^{(0)}<g_{T}^{C}$, a granular metal is in an insulating phase at zero temperature. The metal - insulator transition in three dimensions at $T=0$ occurs at $g_{T}^{C}=(1 / 6 \pi) \ln \left(E_{C} / \delta\right)$, where $E_{C}$ and $\delta$ are the charging energy and the mean energy level spacing in a single grain respectively. At large tunneling conductance, $g_{T}^{(0)}>g_{T}^{C}$, the two different types of conductivity behavior are possible: (i) the high temperature phase, $T>g_{T} \delta$ is characterized by the logarithmic temperature dependence of the conductivity in all dimensions; (ii) the low temperatures phase, $T<g_{T} \delta$ is the universal Granular Fermi liquid phase described in terms of low energy interacting diffusion modes. 
We construct such a universal description of low temperature physical properties of granular metals building on the $\sigma$-model introduced first for the noninteracting dirty metals in Refs. 5, 6 and generalized in Ref. 7 to include the interaction effects. Our approach applies at temperatures $T<g_{T} \delta$, where $\delta$ is the mean energy level spacing in a single grain. The energy scale $g_{T} \delta$ appears naturally as the upper energy cutoff of the effective model, since $\hbar / g_{T} \delta$ is the mean time for the electron to escape from the granule [8].

The main results of our work are as follows: Making use of the effective description of the granular metals in terms of the $\sigma$-model, Eq. (5a), we show that: (i) All the phenomena that are described in terms of the $\sigma$ model including interaction and localization effects, and all the thermodynamic quantities are universal for granular metals. (ii) There are several physical quantities, which, although not directly related to the charges in the $\sigma$-model can, nevertheless, be found from the renormalization group equations describing the flow of charges of the $\sigma$-model. The important example of such a quantity is the tunneling density of states. We summarized our result in Fig. 10 where three distinct phases that one can identify on the basis of our approach are presented: (i) The universal low temperature phase, which we refer to as to the granular Fermi liquid, generalizes naturally the Fermi liquid phase of homogeneously disordered metals. The granular Fermi liquid phase neighbors (ii) the high temperature, $T>g_{T} \delta$, metallic phase governed by the local single grain physics, and (iii) the insulating phase, where $g_{T}^{(0)}<g_{T}^{C}$, characterized by the activation behavior of the conductivity.

Now we turn to the description of our model and the derivation the phase diagram in Fig. 1 We consider a $d$-dimensional array of metallic grains with the Coulomb interaction between electrons. The motion of electrons inside the grains is diffusive, and they can tunnel from grain to grain. We assume that in the absence of the Coulomb interaction, the sample would be a good metal. The system of weakly coupled metallic grains is described by the Hamiltonian

$$
\hat{H}=\hat{H}_{0}+\hat{H}_{i n t}+\sum_{i j} \hat{\psi}^{\dagger}\left(\mathbf{r}_{i}\right) t_{i j} \hat{\psi}\left(\mathbf{r}_{j}\right)
$$

where $t_{i j}$ is the tunneling matrix elements $\left(t_{i j}=t_{j i}\right)$ corresponding to the points of contact $\mathbf{r}_{i}$ and $\mathbf{r}_{j}$ of $i$-th and $j$-th grains. The Hamiltonian $\hat{H}_{0}$ in Eq. (1a) is

$$
\hat{H}_{0}=\sum_{i} \int d^{3} r_{i} \hat{\psi}_{i}^{\dagger}\left[\hat{\mathbf{p}}^{2} / 2 m-\mu+u\left(\mathbf{r}_{i}\right)\right] \hat{\psi}_{i}
$$

with $\mu$ being the chemical potential, it describes noninteracting electrons scattered by random impurity potential $u\left(\mathbf{r}_{i}\right)$. The second term in the right hand side of
Eq. (1a) describes the electron-electron interaction

$$
\hat{H}_{i n t}=\frac{e^{2}}{2} \sum_{i j} \hat{n}_{i} C_{i j}^{-1} \hat{n}_{j},
$$

where $C_{i j}$ is the capacitance matrix and $\hat{n}_{i}=\int d^{3} r_{i} \hat{\psi}_{i}^{\dagger} \hat{\psi}_{i}$ is the electron number operator in the $i$-th grain.

Using Eqs. (II) the $\sigma$-model for granular systems can be derived in a usual way 7, 8, , 9]: we decouple the Coulomb interaction term in Eq. (1C) using the axillary fields $V$, average over disorder introducing $Q$-matrix field $[\underline{6}, \mathbf{7}]$ and expand around the diffusive saddle point. The final expression for the effective low-energy action reads:

$$
\begin{aligned}
S & =-\frac{\pi}{2 \delta} \sum_{i} \operatorname{Tr}\left[\left(\hat{\varepsilon}+V_{i}\right) Q_{i}\right] \\
& -\frac{\pi g_{T}}{8} \sum_{\langle i, j\rangle} \operatorname{Tr}\left[Q_{i} Q_{j}\right]+\frac{1}{T} \sum_{i, j} V_{i}^{*} \frac{C_{i j}}{2 e^{2}} V_{j} .
\end{aligned}
$$

Here the sums are performed over the grain indices, the symbol $\langle\ldots\rangle$ means summation over the nearest neighbors, the trace is taken over spin, replica and Matsubara indices and $\hat{\varepsilon}=i \partial_{\tau}$. The field $V$ in Eq. (2) is a vector in the frequency and replica spaces and the corresponding contraction is assumed: $V^{*} V=\sum_{\omega_{n}, \alpha} V_{\omega_{n}, \alpha}^{*} V_{\omega_{n}, \alpha}$, where $\alpha$ is the replica index and $\omega_{n}=2 \pi T n$ are the bosonic Matsubara frequencies. The $Q$-matrix in Eq. (2) is the matrix in the Matsubara, spin, and replica spaces $Q \rightarrow Q_{\omega_{n_{1}}, \omega_{n_{2}} ; \alpha, \beta}^{a, b}$ subject to constraint $Q^{2}=1$. In addition, each element of the $Q$-matrix is the quaternion, i. e. it can be presented as $Q=q^{i} \tau_{i}$, where $q_{i}$ is the real vector and $\tau_{i}$ are the quaternion matrices [6]. For energies $\varepsilon \ll E_{T}$, where $E_{T}$ is the Thouless energy, the $Q$-matrices in Eq. (2) are coordinate independent within each grain. In what follows we use Hikami-parametrization for $Q$-matrix [10]

$$
Q=\left(\begin{array}{cc}
\sqrt{1-B B^{\dagger}} & B \\
B^{\dagger} & -\sqrt{1-B^{\dagger} B}
\end{array}\right),
$$

where the matrix $B_{\omega_{n_{1}}, \omega_{n_{2}}}$ has non-zero elements only for frequencies $\omega_{n_{1}}>0, \omega_{n_{2}}<0$. Expansion of the $Q$-matrix in powers of the field $B$ in Eq. (2) provides a systematic way to take into account $1 / g_{T}$ corrections.

To derive the low energy $\sigma$-model that provides the universal description of the low temperature phase, $T<$ $g_{T} \delta$, we apply the renormalization group technique to the effective action in Eq. (2). We divide the field $B$ into the slow and fast parts $B=B_{s}+B_{f}$ and define the fast part of the field $B_{\omega_{n_{1}}, \omega_{n_{2}}}$ in a way that it exists either for $\Lambda<\omega_{n_{1}}<\Lambda+d \Lambda$ or $-\Lambda-d \Lambda<\omega_{n_{2}}<-\Lambda$. This procedure (the details will be presented in the forthcoming publication) results in the following renormalization group equation for the tunneling conductance [2]

$$
d g_{T} / d \ln \Lambda=1 /(2 \pi d)
$$


where $d$ is the dimensionality of the granular array. Integration of Eq. (4a) in the energy interval $\left(g_{T} \delta, E_{C}\right)$, where $E_{C}$ is the charging energy for a single grain, results in the following tunneling conductance

$$
g_{T}=g_{T}^{(0)}-(1 / 2 \pi d) \ln \left(E_{C} / \delta\right) .
$$

For energies lower than $g_{T} \delta$ the physics is dominated by the distances that are much larger than the size of a single grain. This allows us to consider the continuum limit of the $\sigma$-model, and arrive at the corresponding final expression for the action:

$$
\begin{aligned}
S & =-\frac{\pi}{2 \delta} \int \operatorname{Tr}\left[(\hat{\varepsilon}+V) Q-\frac{D}{4}(\nabla Q)^{2}\right] \frac{d \mathbf{r}}{a^{d}} \\
& +\frac{1}{T} \int \frac{d \mathbf{r} d \mathbf{r}^{\prime}}{a^{2 d}} \operatorname{Tr}\left[V_{\mathbf{r}}^{*} \frac{C_{\mathbf{r r}^{\prime}}}{2 e^{2}} V_{\mathbf{r}^{\prime}}\right] .
\end{aligned}
$$

Here $a$ is the grain size and the trace is taken over the replica indices. The renormalized diffusion coefficient $D$ in Eq. (5a) is given by

$$
D=g_{T} a^{2} \delta
$$

with $g_{T}$ being the renormalized tunneling conductance from Eq. (4b). Since the effective model (5a) operates with the $Q$-matrices which have only long-range degrees of freedom, it applies, with the appropriate charges and upon the high-energy renormalization, to any disordered metal, including a homogeneously disordered one. Thus, all the information about the granularity of the sample is hidden in the temperature independent renormalization of coefficients of the effective model (5a). The conductivity of the sample is related to the effective diffusion coefficient, $D$ through the usual Einstein relation

$$
\sigma=2 e^{2} D\left(a^{d} \delta\right)^{-1} .
$$

Effective model (5a) together with Eq. (4b) for the renormalized conductance naturally explains the result for the low temperature, $T<g_{T} \delta$, conductivity obtained in Ref. 1. The interaction correction to conductivity has two contributions. The first contribution is temperature independent and is given by

$$
\delta \sigma_{1}=-\sigma_{0} \frac{1}{2 \pi d g_{T}} \ln \left[\frac{E_{C}}{\delta}\right],
$$

where $\sigma_{0}=2 e^{2} g_{T} a^{2-d}$ is the classical Drude conductivity of granular metals. Equation (6b) follows immediately from the renormalization of the tunneling conductance $g_{T}$ in Eq. (4b) and is specific to granular metals. The second contribution to conductivity is temperature dependent and comes from the low energy renormalization of the diffusion coefficient $D$ in the effective model (5a). It coincides with the corresponding correction to conductivity obtained for homogeneously disordered metals in
Ref. 11

$$
\delta \sigma_{2}=\sigma_{0} \begin{cases}\frac{\alpha}{12 \pi^{2} g_{T}} \sqrt{\frac{T}{g_{T} \delta}} & d=3, \\ -\frac{1}{4 \pi^{2} g_{T}} \ln \frac{g_{T} \delta}{T} & d=2, \\ -\frac{\beta}{4 \pi} \sqrt{\frac{\delta}{T g_{T}}} & d=1 .\end{cases}
$$

where $\alpha \approx 1.83$ and $\beta \approx 3.13$ are the numerical constants.

Although the tunneling density of states is not directly related to the charges in the $\sigma$-model, it can, nevertheless, be found from the renormalization group equations describing the flow of charges of the $\sigma$-model. Since at low temperatures the flow of coupling constants of the $\sigma$-model of granular metals is determined by the same renormalization group equations as in the case of homogeneously disordered metals, one arrives at the important conclusion that the tunneling density of states has a multiplicative structure:

$$
\nu / \nu_{0}=\nu_{h} \nu_{l},
$$

where $\nu_{0}$ is the density of states of non-interacting electrons, $\nu_{h}$ is the contribution to the density of sates that comes from high energies, $\varepsilon>g_{T} \delta$, while $\nu_{l}$ is the contribution from low energies, $\varepsilon<g_{T} \delta$ which up to the proper renormalization of all constants coincides with the corresponding result for the density of states of disordered homogeneous metals. As an application of Eq. (7) let us consider the density of states of granular films. The low energy contribution, $\nu_{l}$ in Eq. (7), is

$$
\nu_{l}=\exp \left[-\frac{1}{16 g_{T} \pi^{2}} \ln \frac{g_{T} \delta}{T} \ln \frac{g_{T} E_{C}^{4}}{T \delta^{3}}\right],
$$

whereas the "high energy" part of the tunneling density of states, $\nu_{h}$ in Eq. (7), is temperature independent at $T<g_{T} \delta$ and for granular films is given by

$$
\nu_{h}=\left[\frac{E_{C}}{\delta}\right]^{1 / \pi}\left[1-\frac{\ln \left(E_{C} / \delta\right)}{4 \pi g_{T}^{(0)}}\right]^{4 g_{T}^{(0)}}
$$

At large tunneling conductance, $g_{T} \gg 1$, the result for the low energy contribution, $\nu_{l}$, in Eq. 8a coincides with the perturbative result for the density of states of granular metals obtained in Ref. [1].

Having described the effects of electron-electron interaction on the transport properties of granular metals, we now turn to quantum- (or weak localization) corrections to the conductivity [12]. In the leading order in the inverse tunneling conductance, $1 / g_{T}$, interaction and weak localization corrections can be considered independently. As usual, the weak localization correction $\delta \sigma_{W L}$ is defined by the following expression

$$
\delta \sigma_{W L}=-\frac{2}{\pi} e^{2} g_{T} \int C(0, \mathbf{q}) \frac{d^{d} q}{(2 \pi)^{d}},
$$

where $C(0, \mathbf{q})$ is the Cooperon propagator which in the absence of electron-electron interaction is given by 
$C(\omega, \mathbf{q})=\left(D \mathbf{q}^{2}-i \omega\right)^{-1}$ with $D$ being the effective diffusion coefficient in Eq. (5b). For $2 D$ and $1 D$ samples it is important to take into account dephasing effects since Eq. (9) diverges. Dephasing time $\tau_{\phi}$ may be obtained from the effective model (5a) straightforwardly using the corresponding results for homogeneously disordered metals 13 with the proper effective diffusion coefficient, $D=g_{T} a^{2} \delta$. The final result for the weak localization corrections reads

$$
\frac{\delta \sigma_{W L}}{\sigma_{0}}=-\frac{1}{4 \pi^{2} g_{T}} \ln \left(\frac{g_{T}^{2} \delta}{T}\right),
$$

for granular films, and

$$
\frac{\delta \sigma_{W L}}{\sigma_{0}}=-\frac{1}{2 \pi g_{T}}\left(\frac{g_{T}^{2} \delta}{T}\right)^{1 / 3}
$$

for granular wires. We notice that the quantum interference corrections $\delta \sigma_{W L}$ may be easily suppressed by applying relatively weak magnetic field such that the main temperature dependence of the conductivity comes from electron-electron interaction effects Eqs. (6b) and (6c).

In conclusion, we have derived the low energy $\sigma$-model that provides a universal description of the low temperature phase of granular metals and, more generally, of any disordered conducting medium. This model explains a striking similarity of the low-temperature transport behaviors of different disordered conductors as being governed by the same long wave electronic diffusion modes. The proposed model enables one to derive the low energy properties of granular metals from the corresponding characteristics of disordered homogeneous metals. We demonstrated the power of the developed approach by finding the density of states, the interaction and localization corrections to the conductivity of granular metals.

We thank K. Efetov, L. Glazman, D. Khmel'nitskii and A. Koshelev for illuminating discussions. This work was supported by the U. S. Department of Energy, Office of Science through contract No. W-31-109-ENG-38.

[1] I. S. Beloborodov, K. B. Efetov, A. V. Lopatin and V. M. Vinokur, Phys. Rev. Lett 91, 246801 (2003).

[2] K. B. Efetov and A. Tschersich, Europhys. Lett. 59, 114, (2002); Phys. Rev. B 67, 174205 (2003).

[3] A. Gerber et al, Phys. Rev. Lett 78, 4277 (1997).

[4] R. W. Simon et al, Phys. Rev. B 36, 1962 (1987).

[5] F. Wegner, Z. Phys. B 35, 207 (1979).

[6] K. B. Efetov, A. I. Larkin and D. E. Khmel'nitskii, Zh. Eksp. Teor. Fiz. 79, 1120 (1980) [Sov. Phys. JETP 52, 568 (1980)].

[7] M. Finkelstein, Electron liquid in Disordered Conductors, edited by I. M. Khalatnikov, Soviet Scientific Reviews Vol. 14 (Harwood, London, 1990).

[8] I. S. Beloborodov, K. B. Efetov, A. Altland and F. W. J. Hekking, Phys. Rev. B 63, 115109 (2001).

[9] A. V. Andreev and I. S. Beloborodov, Phys. Rev. B 69, 081406(R) (2004).

[10] S. Hikami, Phys. Rev. B 24, 2671 (1981).

[11] B. L. Altshuler and A. G. Aronov, in Electron-Electron Interaction in Disordered Systems, ed. by A. L. Efros and M. Pollak, North-Holland, Amsterdam (1985).

[12] L. P. Gorkov, A. L. Larkin and D. E. Khmel'nitskii, Pis'ma Zh. Eksp. Teor. Fiz. 30, 248 (1979) [Sov. Phys. JETP Lett. 30, 228 (1979)].

[13] B. L. Altshuler, A. G. Aronov and D. E. Khmel'nitskii, J. Phys. C 15, 7367 (1982). 